\title{
Prevention of post-ERCP pancreatitis: NSAID or pancreatic stent or both?
}

\section{다)(우우}

\author{
Authors \\ R. L. J. van Wanrooij, J. E. van Hooft
}

\author{
Corresponding author \\ Jeanin E van Hooft, MD, PhD, MBA, Amsterdam UMC - \\ gastroenterology, meibergdreef 9 Amsterdam 1105AZ, \\ Netherlands \\ Fax: +0205663556 \\ j.e.vanhooft@amc.uva.nl
}

Endoscopic retrograde cholangiopancreaticography (ERCP) is a widely used endoscopic procedure that facilitates therapeutic interventions in the pancreaticobiliary system. From $3 \%$ to $15 \%$ of patients develop post-ERCP pancreatitis (PEP), which leads to significant morbidity and in some cases even mortality [1] PEP results in a financial burden for health care systems and in the United States alone, management of it annually costs over \$200 million [1].

Currently two prophylactic interventions have shown to be effective in preventing PEP: placement of a pancreatic stent and administration of rectal nonsteroidal anti-inflammatory drugs (NSAIDs). Both interventions attempt to prevent development of PEP in different ways. While pancreatic stents overcome the outflow obstruction of pancreatic juices due to iatrogenic edema of the papilla, anti-inflammatory drugs attenuate the early inflammatory response to prevent initiation of the inflammatory cascade. The question that follows is whether these two interventions, in theory complementary, exert a synergistic effect or whether one of these measures suffices. However, so far, no head-to-head trials have been published to answer this question. To this end a network meta-analysis, including 29 studies indirectly comparing various prophylactic strategies, was performed which suggested superiority of rectal NSAID over a pancreatic stent in prevention of PEP [2]. The combination of pancreatic stent and rectal NSAID was not more effective in comparison to rectal NSAID alone [2].

In addition, a post hoc analysis of a randomized controlled trial evaluating the effect of rectal NSAID in PEP prevention even suggested that the combination of a pancreatic stent and rectal NSAID was in fact worse than rectal NSAID alone [3]. An explanation for this observation could be that the additional manipulation in attempt to place a pancreatic stent leads to pancreatic duct injury, while the best strategy might be to avoid any unnecessary manipulation.

In this issue of Endoscopy International Open, Philip et al. nicely show that in patients in whom the pancreatic duct was inadvertently cannulated, placement of a pancreatic stent significantly lowered incidence of PEP (12.6\%) in comparison to the control group without pancreatic stent (25\%) [4]. The results of this study underline the use of pancreatic stents in patients who were exposed to manipulation of the pancreatic duct and therefore carried an increased risk of developing PEP.

In patients with an average risk for PEP, the role of prophylactic pancreatic stents is much less clear, as pancreatic stents are associated with various problems. First, an additional x-ray followed by an endoscopy in $60 \%$ of patients to remove the pancreatic stents after 2 weeks leads to extra burden to patients and additional cost [3]. Second, pancreatic stents can inflict (permanent) damage to the pancreatic duct, causing stenosis and recurrent pancreatitis, especially when the stent migrates proximally [5]. Third, in $5 \%$ to $20 \%$ of patients, placement of a pancreatic stent is not successful, thereby actually increasing risk of PEP, in patients without rectal NSAID up to $35 \%$ [6]. Noteworthy, this negative effect of failed pancreatic stent placement appears to be fully attenuated by rectal NSAID [6].

In the study by Philip et al, patients were denied rectal NSAID as the authors argue that the study results from rectal NSAIDs in preventing PEP were not uniformly positive and largely based on studies in high-risk patients [4]. The latter argument seems inconsistent as their study also included high-risk patients by definition (insertion of guidewire in pancreatic duct) which is illustrated by the substantial incidence of PEP found in their study. Regarding the efficacy of NSAIDs, there are considerable data that do support use of rectal NSAIDs to prevent PEP in 
high-risk patients, whereas the evidence in average-risk patients is indeed much less robust [7]. An important factor to consider is that rectal NSAIDs exert a better protective effect when administered before instead of after ERCP, probably due to optimal pharmacological availability [8]. Because risk of PEP is largely determined by procedure-related events that are unknown beforehand, in our opinion, patients without a contraindication for NSAIDs should not be denied such an effective prophylactic measure that is inexpensive and carries a mild sideeffect profile, which is in agreement with the guideline of the European Society of Gastrointestinal Endoscopy [9]. Finally, it should be mentioned that periprocedural hyperhydration with Ringer's lactate also shows promise in prevention of PEP [10]. Data are still sparse but larger randomized controlled trials are underway, including the Fluyt trial (NL5020) that compares hyperhydration and rectal NSAID to rectal NSAID only in 826 moderate- to high-risk patients [11].

Considering all of this, we adhere to pre-procedural administration of rectal NSAIDs in all patients without contraindications, and place pancreatic stents only in patients at high risk for PEP, especially when a guidewire has already been inadvertently placed in the pancreatic duct. Nevertheless, it is still a subject of concern how much manipulation during placement of a pancreatic stent is within reason and outweighs the increased risk of PEP caused by this manipulation. So, in cases in which the position of the pancreatic duct guidewire is lost, how many attempts does the endoscopist allow him or herself to regain position?

Future research will show whether periprocedural hyperhydration is another effective measure in prevention of PEP, and if so, whether it should be used in combination with NSAID or pancreatic stent or both.

\section{Competing interests}

None
References

[1] Kochar B, Akshintala VS, Afghani E et al. Incidence, severity, and mortality of post-ERCP pancreatitis: a systematic review by using randomized, controlled trials. Gastrointest Endosc 2015; 81: 143 149 e9

[2] Akbar A, Abu Dayyeh BK, Baron TH et al. Rectal nonsteroidal anti-inflammatory drugs are superior to pancreatic duct stents in preventing pancreatitis after endoscopic retrograde cholangiopancreatography: a network meta-analysis. Clin Gastroenterol Hepatol 2013; 11: $778-783$

[3] Elmunzer B], Higgins PD, Saini SD et al. Does rectal indomethacin eliminate the need for prophylactic pancreatic stent placement in patients undergoing high-risk ERCP? Post hoc efficacy and cost-benefit analyses using prospective clinical trial data Am J Gastroenterol 2013; 108: 410-415

[4] Philip VPA, Epstein A, Hapfelmeier A et al. Pancreatic stenting to prevent post-ERCP pancreatitis: a randomized multicenter trial. Endosc Int Open 2019; 07: E860 -E868

[5] Bakman YG, Safdar K, Freeman ML. Significant clinical implications of prophylactic pancreatic stent placement in previously normal pancreatic ducts. Endosc 2009; 41: 1095-1098

[6] Choksi NS, Fogel EL, Cote GA et al. The risk of post-ERCP pancreatitis and the protective effect of rectal indomethacin in cases of attempted but unsuccessful prophylactic pancreatic stent placement. Gastrointest Endosc 2015; 81: $150-155$

[7] Yang C, Zhao Y, Li W et al. Rectal nonsteroidal anti-inflammatory drugs administration is effective for the prevention of post-ERCP pancreatitis: An updated meta-analysis of randomized controlled trials. Pancreatology 2017; 17: 681-688

[8] Luo $\mathrm{H}$, Wang $\mathrm{X}$, Zhang $\mathrm{R}$ et al. Rectal indomethacin and spraying of duodenal papilla with epinephrine increases risk of pancreatitis following endoscopic retrograde cholangiopancreatography. Clin Gastroenterol Hepatol 2018: doi:10.1016/j.cgh.2018.10.043

[9] Dumonceau JM, Andriulli A, Elmunzer B] et al. Prophylaxis of postERCP pancreatitis: European Society of Gastrointestinal Endoscopy (ESGE) Guideline - updated June 2014. Endoscopy 2014; 46: 799 815

[10] Mok SRS, Ho HC, Shah P et al. Lactated Ringer's solution in combination with rectal indomethacin for prevention of post-ERCP pancreatitis and readmission: a prospective randomized, double-blinded, placebo-controlled trial. Gastrointest Endosc 2017; 85: 1005-1013

[11] Smeets X, da Costa DW, Fockens P et al. Fluid hydration to prevent post-ERCP pancreatitis in average- to high-risk patients receiving prophylactic rectal NSAIDs (FLUYT trial): study protocol for a randomized controlled trial. Trials 2018; 19: 207 